\title{
Fundamental Approach for Analysis of Dynamic Characteristics of Fixtures
}

\author{
Vitalii Ivanov $^{1, *}$, Ivan Pavlenko ${ }^{2}$ \\ ${ }^{1}$ Sumy State University, 2 Rymskogo-Korsakova St., Sumy, 40007, Ukraine, ivanov@tmvi.sumdu.edu.ua \\ ${ }^{2}$ Sumy State University, 2 Rymskogo-Korsakova St., Sumy, 40007, Ukraine, i.pavlenko@ omdm.sumdu.edu.ua
}

\begin{abstract}
Present work is aimed at research of locating and clamping process of prismatic parts in fixtures during the machining on drilling-milling-boring machines. The fundamental approach for developing mathematical modeling of the system "fixture - workpiece" has been proposed. It allows to determine critical frequencies of functional elements oscillations and amplitude-frequency response characteristics, as well as dynamic component of fixture deformations at operating frequency of the machining process. The developed mathematical model is evaluated by numerical calculations of spectrum of critical frequencies considering the stiffness of cutting tool. The further researches are directed on the development of the methodology of calculation of the system "fixture - workpiece" by computer means of numerous realization and development of practical recommendations regarding its use during fixture design.
\end{abstract}

Keywords: functional element, stiffness, free oscillations, eigenfrequency, forced oscillations, cutting force, amplitude-frequency response.

Received on 02 December 2017, accepted on 13 January 2018, published on 20 March 2018

Copyright (C) 2018 Ivanov V. et al., licensed to EAI. This is an open access article distributed under the terms of the Creative Commons Attribution licence (http://creativecommons.org/licenses/by/3.0/), which permits unlimited use, distribution and reproduction in any medium so long as the original work is properly cited.

doi: 10.4108/eai.20-3-2018.154366

\section{Introduction}

Fixtures are used for ensuring the precise locating and reliable clamping of work-pieces during the machining on the metal-cutting machines and are an integral part of the closed-loop technological system "machine tool - fixture cutting tool - work-piece". Fixtures considerably effect on the output of the competitive products, ensuring the steady state accuracy and quality of the work surfaces, sufficient stiffness of the technological system, flexibility of production and initial cost of the final product. It is confirmed by the percentage number of fixtures that makes

$70-80 \%$ from the total quantity of tooling, $10-20 \%$ of the total cost of manufacturing system, $80-90 \%$ of the total time for production planning, $40 \%$ of the rejected parts after the machining due to imperfection fixture design, $70 \%$ of new fixtures are the modification of the existing [1-5].

*Corresponding author. Email: ivanov@tmvi.sumdu.edu.ua
Besides, most of existing methods of calculation do not include the dynamic character of the cutting process and interaction of the workpiece with the elements of fixture. Therefore, the problem of designing of fixtures is very urgent and relates to the basic multidisciplinary scientific and practical tasks on ensuring the stable locating of workpiece in the fixture during the machining process of the surfaces of parts.

\section{Literature Review}

At present, different approaches to the design and research of fixture for carrying out the manufacturing operations of machining of parts of different types are being developed. With the purpose of determining of the present state of researches in fixture designing and determining of the urgency of the research, the basic approaches to the designing of fixture from the point of view of interaction between fixture and workpiece have been considered. 
Friction between elements of fixture and workpiece has been researched and have been determined the deformations, which appear in the points of contact [6]. The methodology of modeling of the system "fixture - workpiece" was developed and the influence of the previous load from the impact of clamping and cutting forces on the error of the work surface was determined [7]. The methodology of analysis of stability of the system "fixture - workpiece" has been developed and calculation of the minimum clamping force, required for machining process, has been presented; also, the influence of sequence of workpiece clamping has been researched [8]. The influence of modes of cutting and fixture compliance on the workpiece stability was analytically calculated and researched [9]. The simplified analytical model of the contact interaction between clamping elements of fixture and workpiece and the developed finite element model allows evaluating the contact deformation in contact points between clamping elements and workpiece were presented [10]. The finite element model of determination the stability of workpiece locating in fixture and the methodology of optimization of the previous loading were developed [11]. The mathematical model of fixture interaction with workpiece and analysis of deterministic positioning of fixture was developed [12]. Many researches in fixture accuracy and analysis of the fixture stability were realized, based on the consideration of two-dimensional problem and introduction of "operative factor", which considers the friction forces [13]. The methodology on determination of points and forces of clamping for ensuring the stable locating of workpiece in fixture has been developed [14]. The methodology of control of clamping force considering the contact interaction between workpiece and clamping elements by means of the methods of nonlinear programming was presented [15]. The problem of the temporariness of the stability of fixture locating considering the restriction of the force and direction of its action in the system "fixture - workpiece" has been researched [16]. The influence of material removal on the dynamic state of the system "fixture - workpiece" was considered as opposed to quasi-static approach of predecessors [17]. An essential experience of research of mechanical system "fixture - workpiece" has been obtained in terms of ensuring the condition of workpiece stability in fixture [18], [19].

Considering that the existing information on determine the contact points of functional elements of fixture, which are locating and clamping elements, is of advisory character and do not base on the analytical researches; and basing on that fact that the existing methodologies of calculation of clamping forces do not include the condition of stability of workpiece and provide the unreasonably overestimated value, performing of the researches is the relevant objective.

The present work is focused on locating and clamping of prismatic parts in fixtures during the machining on drillingmilling-boring machines. The characteristic feature of this scheme is that three flat surfaces are used as a datum (3-2-1 locating chart), ensuring the stability, convenience of locating and preventing of oscillations during the machining [20].
The goal of the proposed work is the development of the fundamental approach for mathematical modeling of the system "fixture - workpiece" based on manufacturing features of the process of locating and clamping of the workpiece in the fixture considering dynamic analysis of the workpiece in fixture under the effect of spatial system of cutting and clamping forces.

\section{Materials and Methods}

\subsection{Dynamic equation}

Consider the conservative mechanical system, which consists from workpiece, clamped in 6 supports $1,2, \ldots, 6$ in the global orthogonal coordinate system XYZ with the center in the point $\mathrm{O}$ (Fig. 1).

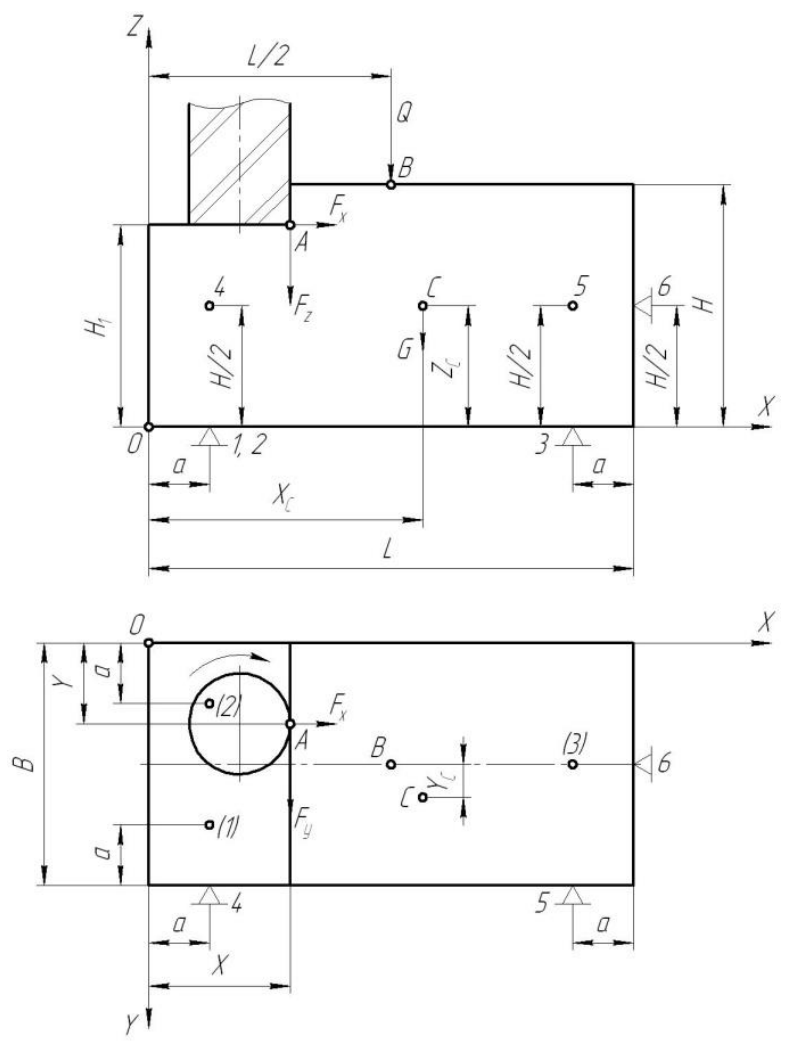

Figure 1. Calculation scheme of the mechanical system "fixture - workpiece"

External active forces are:

- components of the cutting force Fx, Fy and Fz, applied in the point $\mathrm{A}(\mathrm{X}, \mathrm{Y}, \mathrm{H} 1)$;

- clamping force $\mathrm{Q}$, applied in the point $\mathrm{B}(\mathrm{L} / 2, \mathrm{~B} / 2, \mathrm{H})$;

- weight of the workpiece $\mathrm{G}$, applied in the weight center $\mathrm{C}(\mathrm{XC}, \mathrm{YC}, \mathrm{ZC})$.

Consider the space movement of the workpiece, calculation scheme of which is presented on the Fig. 2. 


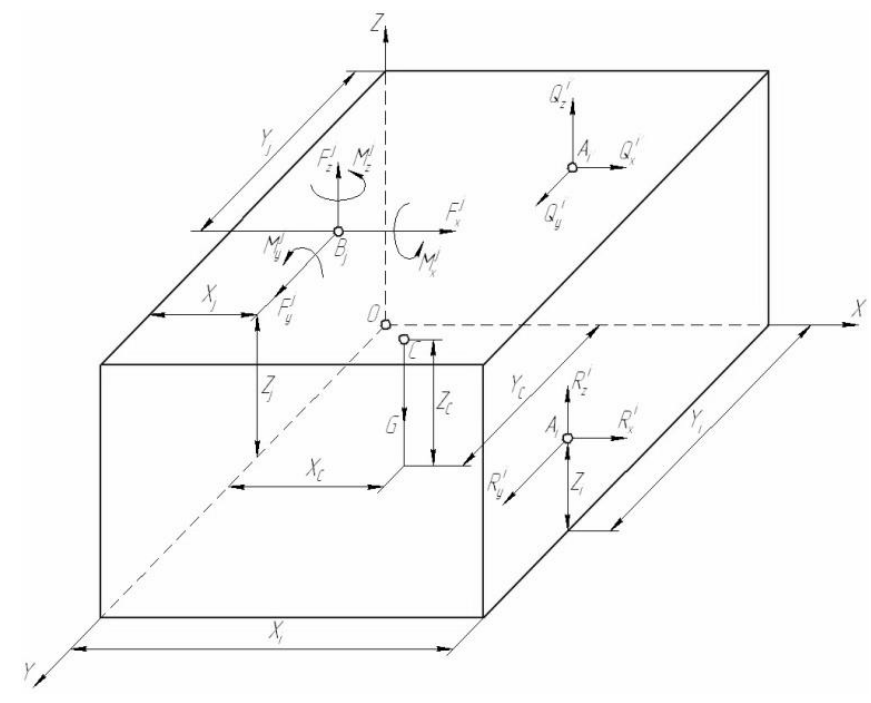

a

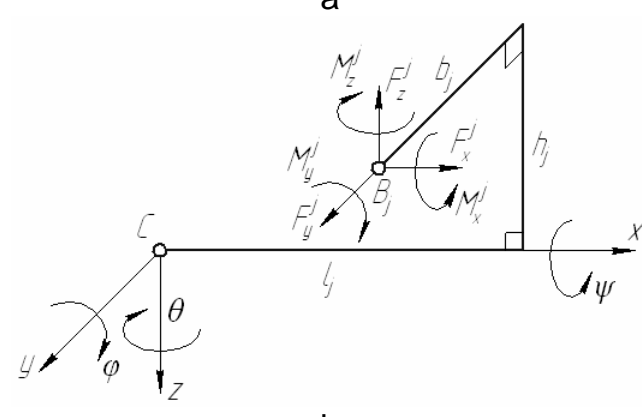

b

Figure 2. Calculation scheme (a) and the system of dynamic components of cutting forces (b)

Displacement of xi, yi, zi of the supporting surfaces of work-piece in the fixture regarding the stationary location, that is characterized by static displacement, determined because of static calculation, could be expressed by geometric relation through 6 independent parameters degrees of freedom of workpiece (Fig. 3):

- components of displacement of the center of weights $\mathrm{xC}, \mathrm{yC}, \mathrm{zC}$;

- rotation angles $\varphi, \psi, \theta$ of three mutually perpendicular planes $y-z, x-z, x-y$ around axes $x, y, z$ of the local coordinate system, which pass through the center of the weight of workpiece $\mathrm{C}$ :

$$
\begin{aligned}
& x_{i}=x_{C}-\Delta x_{i}^{\prime}+\Delta x_{i}^{\prime \prime} ; \\
& y_{i}=y_{C}+\Delta y_{i}^{\prime}+\Delta y_{i}^{\prime \prime \prime} \\
& z_{i}=z_{C}+\Delta z_{i}^{\prime \prime}+\Delta z_{i}^{\prime \prime \prime},
\end{aligned}
$$

where $\Delta x i /, \Delta x i / /, \Delta$ yi /, $\Delta$ yi ///, $\Delta$ zi //, $\Delta$ zi // - additional displacements of sup-ports through the rotation angles $\varphi, \psi$, $\theta$, determined by trigonometric ratios:

$$
\begin{aligned}
& \Delta x_{i}^{\prime}=b_{i} \sin \theta+l_{i}(1-\cos \theta) ; \\
& \Delta y_{i}^{\prime}=l_{i} \sin \theta-b_{i}(1-\cos \theta) ; \\
& \Delta x_{i}^{\prime \prime}=h_{i} \sin \varphi-l_{i}(1-\cos \varphi) ; \\
& \Delta z_{i}^{\prime \prime}=l_{i} \sin \varphi+h_{i}(1-\cos \varphi) ; \\
& \Delta y_{i}^{\prime \prime \prime}=h_{i} \sin \psi-b_{i}(1-\cos \psi) ; \\
& \Delta z_{i}^{\prime \prime \prime}=b_{i} \sin \psi+h_{i}(1-\cos \psi) .
\end{aligned}
$$

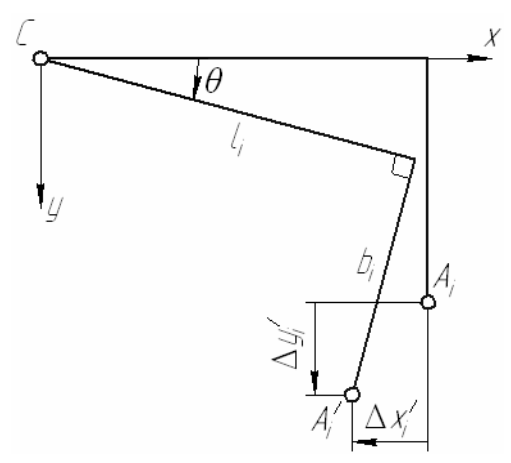

a
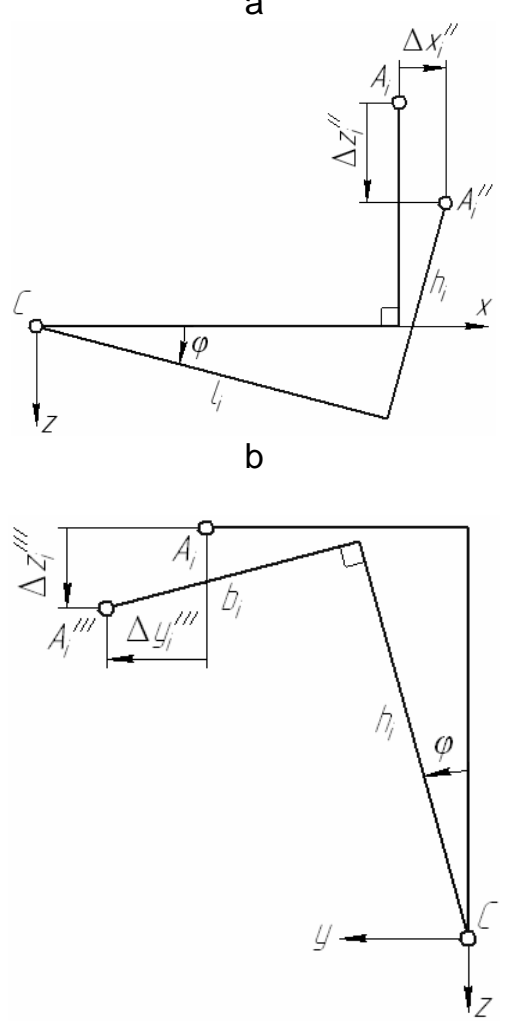

C

Figure 3. Geometric rations for determination the additional displacements: $\Delta \mathrm{x}^{\prime}, \Delta \mathrm{y}_{\mathrm{i}}{ }^{\prime}(\mathrm{a}) ; \Delta \mathrm{x}^{\prime \prime}, \Delta \mathrm{zi}^{\prime \prime}(\mathrm{b})$; $\Delta \mathrm{yi}^{\prime \prime \prime}, \Delta \mathrm{zi}^{\prime \prime \prime}(\mathrm{c})$

Parameters $\mathrm{l}_{\mathrm{i}}, \mathrm{b}_{\mathrm{i}}, \mathrm{h}_{\mathrm{i}}$ are local coordinates of locating elements $1,2, \ldots, 6$, connected with global coordinates $\mathrm{X}_{\mathrm{i}}$, $\mathrm{Y}_{\mathrm{i}}, \mathrm{Z}_{\mathrm{i}}$ by the following relations: 


$$
l_{i}=X_{i}-X_{C} ; \quad b_{i}=Y_{i}-Y_{C} ; \quad h_{i}=Z_{i}-Z_{C} .
$$

For relatively small values of deformation of supports the statements (2) could be expanded into a Maclaurin series, holding members of the first order of smallness:

$$
\begin{aligned}
\Delta x_{i}^{\prime}=b_{i} \theta, & \Delta x_{i}^{\prime \prime}=h_{i} \varphi ; \\
\Delta y_{i}^{\prime}=l_{i} \theta, & \Delta y_{i}^{\prime \prime \prime}=h_{i} \psi ; \\
\Delta z_{i}^{\prime \prime}=l_{i} \varphi, & \Delta z_{i}^{\prime \prime \prime}=b_{i} \psi .
\end{aligned}
$$

Herewith, the components of displacements of supports (1) take the form:

$$
\begin{aligned}
& x_{i}=x_{C}-b_{i} \theta+h_{i} \varphi ; \\
& y_{i}=y_{C}+l_{i} \theta+h_{i} \psi ; \\
& z_{i}=z_{C}+l_{i} \varphi+b_{i} \psi .
\end{aligned}
$$

The space movement of the workpiece in the fixture could be described by means of the theorem on movement of the center of weight and the theorem on replacement of the angular momentum of mechanical system in the projections on the axis of the local coordinate system xyz:

$$
\left\{\begin{array}{l}
m \ddot{x}_{C}=\Sigma F_{s x}^{i} ; \quad m \ddot{y}_{C}=\Sigma F_{s y}^{i} ; \quad m \ddot{z}_{C}=\Sigma F_{s z}^{i} ; \\
J_{C x} \ddot{\psi}=\Sigma M_{s x}^{i} ; \quad J_{C y} \ddot{\varphi}=\Sigma M_{s y}^{i} ; \quad J_{C z} \ddot{\theta}=\Sigma M_{s z}^{i},
\end{array}\right.
$$

where $\mathrm{m}$ - weight of workpiece; $\mathrm{J}_{\mathrm{Cx}}, \mathrm{J}_{\mathrm{Cy}}, \mathrm{J}_{\mathrm{Cz}}$ - inertia moments of workpiece relative to the axis, which pass through the center of weight; $\ddot{x}_{C}, \ddot{y}_{C}, \ddot{z}_{C}$ - projections of acceleration of the center of weight on the coordinate system; $\ddot{\psi}, \ddot{\varphi}, \ddot{\theta}$ - angular acceleration of the workpiece relatively to the coordinate axes.

Components of the spring forces and their moments (Fig. $2 \mathrm{~b}$ ) relative to the coordinate axes considering the relations (5) are defined by the statements (7). Statements (7) allow presenting the equations set in other form (8).

$$
\begin{aligned}
& \Sigma F_{s x}^{i}=-\Sigma k_{x}^{i} x_{i}=-\Sigma k_{x}^{i}\left(x_{C}-b_{i} \theta+h_{i} \varphi\right) ; \\
& \Sigma F_{s y}^{i}=-\Sigma k_{y}^{i} y_{i}=-\Sigma k_{y}^{i}\left(y_{C}+l_{i} \theta+h_{i} \psi\right) ; \\
& \Sigma F_{s z}^{i}=-\Sigma k_{z}^{i} z_{i}=-\Sigma k_{z}^{i}\left(z_{C}+l_{i} \varphi+b_{i} \psi\right) ; \\
& \Sigma M_{s x}^{i}=\Sigma\left(-F_{s y}^{i} h_{i}-F_{s z}^{i} b_{i}\right)=-\Sigma\left[k_{y}^{i} h_{i}\left(y_{C}+l_{i} \theta+h_{i} \psi\right)+k_{z}^{i} b_{i}\left(z_{C}+l_{i} \varphi+b_{i} \psi\right)\right] \\
& \Sigma M_{s y}^{i}=\Sigma\left(-F_{s x}^{i} h_{i}-F_{s z}^{i} l_{i}\right)=-\Sigma\left[k_{x}^{i} h_{i}\left(x_{C}-b_{i} \theta+h_{i} \varphi\right)+k_{z}^{i} l_{i}\left(z_{C}+l_{i} \varphi+b_{i} \psi\right)\right] \\
& \Sigma M_{s x}^{i}=\Sigma\left(F_{s x}^{i} b_{i}-F_{s y}^{i} l_{i}\right)=-\Sigma\left[-k_{x}^{i} b_{i}\left(x_{C}-b_{i} \theta+h_{i} \varphi\right)+k_{y}^{i} l_{i}\left(y_{C}+l_{i} \theta+h_{i} \psi\right)\right] \\
& \left\{\begin{array}{l}
m \ddot{x}_{C}+\Sigma k_{x}^{i} x_{C}+\Sigma k_{x}^{i} h_{i} \varphi-\Sigma k_{x}^{i} b_{i} \theta=F_{x} ; \\
m \ddot{y}_{C}+\Sigma k_{y}^{i} y_{C}+\Sigma k_{y}^{i} h_{i} \psi+\Sigma k_{y}^{i} l_{i} \theta=F_{y} ; \\
m \ddot{z}_{C}+\Sigma k_{z}^{i} z_{C}+\Sigma k_{z}^{i} b_{i} \psi+\Sigma k_{z}^{i} l_{i} \varphi=F_{z} ; \\
J_{C x} \ddot{\psi}+\Sigma k_{y}^{i} h_{i} y_{C}+\Sigma k_{z}^{i} b_{i} z_{C}+\Sigma\left(k_{y}^{i} h_{i}^{2}+k_{z}^{i} b_{i}^{2}\right) \psi+\Sigma k_{z}^{i} l_{i} b_{i} \varphi+\Sigma k_{y}^{i} l_{i} h_{i} \theta=M_{x} ; \\
J_{C y} \ddot{\varphi}+\Sigma k_{x}^{i} h_{i} x_{C}+\Sigma k_{z}^{i} l_{i} z_{C}+\Sigma k_{z}^{i} l_{i} b_{i} \psi+\Sigma\left(k_{x}^{i} h_{i}^{2}+k_{z}^{i} l_{i}^{2}\right) \varphi-\Sigma k_{x}^{i} b_{i} h_{i} \theta=M_{y} ; \\
J_{C z} \ddot{\theta}-\Sigma k_{x}^{i} b_{i} x_{C}+\Sigma k_{y}^{i} l_{i} y_{C}+\Sigma k_{y}^{i} l_{i} h_{i} \psi-\Sigma k_{x}^{i} b_{i} h_{i} \varphi+\Sigma\left(k_{x}^{i} b_{i}^{2}+k_{y}^{i} l_{i}^{2}\right) \theta=M_{z},
\end{array}\right.
\end{aligned}
$$

where $\mathrm{m}$ - weight of the workpiece; $\mathrm{J}_{\mathrm{Cx}}, \mathrm{J}_{\mathrm{Cy}}, \mathrm{J}_{\mathrm{Cz}}$ - inertia moments of workpiece relative to the axis, which pass through the center of weight; $\ddot{x}_{C}, \ddot{y}_{C}, \ddot{z}_{C}$-projections of acceleration of the center of weight on the coordinate system; $\ddot{\psi}, \ddot{\varphi}, \ddot{\theta}-$ angular acceleration of the workpiece relatively to the coordinate axes; $F_{x}, F_{y}, F_{z}$ - dynamical components of the cutting forces (Fig. 2 a).

Thus, the mathematical model of dynamic analysis of the workpiece in fixture is described by the system of differential equations of the 12-th order relative to 6 changeable in time independent parameters $\mathrm{x}_{\mathrm{C}}, \mathrm{y}_{\mathrm{C}}, \mathrm{z}_{\mathrm{C}}, \varphi$, $\psi, \theta$.

\subsection{Research of the free oscillations}

For the case of zero value of forces and cutting moments, the equations set (8) in matrix form takes the following form:

$$
[M]\left\{\ddot{X}_{C}\right\}+[K]\{X\}=\{0\},
$$

where $\{0\}=\{0,0, \ldots, 0\}^{\mathrm{T}}-$ zero vector-column of an external action; $\{\mathrm{X}\}=\left\{\mathrm{x}_{\mathrm{C}}, \mathrm{y}_{\mathrm{C}}, \mathrm{z}_{\mathrm{C}}, \varphi, \psi, \theta\right\}^{\mathrm{T}}$ - vectorcolumn of desired displacement; $[\mathrm{M}],[\mathrm{K}]-$ symmetric matrix of inertia and stiffness: 


$$
\begin{gathered}
{[M]=[M]^{T}=\left[\begin{array}{cccccc}
m & 0 & 0 & 0 & 0 & 0 \\
0 & m & 0 & 0 & 0 & 0 \\
0 & 0 & m & 0 & 0 & 0 \\
0 & 0 & 0 & J_{C x} & 0 & 0 \\
0 & 0 & 0 & 0 & J_{C y} & 0 \\
0 & 0 & 0 & 0 & 0 & J_{C z}
\end{array}\right] ;} \\
{[K]=[K]^{T}=\left[\begin{array}{cccccc}
k_{x x} & 0 & 0 & 0 & k_{x \varphi} & -k_{x \theta} \\
0 & k_{y y} & 0 & k_{y \psi} & 0 & k_{y \theta} \\
0 & 0 & k_{z z} & k_{z \psi} & k_{z \varphi} & 0 \\
0 & k_{y \psi} & k_{z \psi} & k_{\psi \psi} & k_{\psi \varphi} & k_{\psi \theta} \\
k_{x \varphi} & 0 & k_{z \varphi} & k_{\psi \varphi} & k_{\varphi \varphi} & -k_{\varphi \theta} \\
-k_{x \theta} & k_{y \theta} & 0 & k_{\psi \theta} & -k_{\varphi \theta} & k_{\theta \theta}
\end{array}\right] .}
\end{gathered}
$$

Stiffness matrix $[\mathrm{K}]$ includes 6 consolidated coefficients of the direct stiffness

$$
\begin{aligned}
& k_{x x}=\Sigma k_{x}^{i} ; \quad k_{y y}=\Sigma k_{y}^{i} ; \\
& k_{z z}=\Sigma k_{z}^{i} ; \\
& k_{\psi \psi}=\Sigma\left(k_{y}^{i} h_{i}^{2}+k_{z}^{i} b_{i}^{2}\right) ; \\
& k_{\varphi \varphi}=\Sigma\left(k_{x}^{i} h_{i}^{2}+k_{z}^{i} l_{i}^{2}\right) ; \\
& k_{\theta \theta}=\Sigma\left(k_{x}^{i} b_{i}^{2}+k_{y}^{i} l_{i}^{2}\right)
\end{aligned}
$$

and 9 consolidated coefficients of the cross stiffness:

$$
\begin{aligned}
& k_{x \varphi}=\Sigma k_{x}^{i} h_{i} ; k_{x \theta}=\Sigma k_{x}^{i} b_{i} ; k_{y \psi}=\Sigma k_{y}^{i} h_{i} ; \\
& k_{y \theta}=\Sigma k_{y}^{i} l_{i} ; k_{z \psi}=\Sigma k_{z}^{i} b_{i} ; k_{z \varphi}=\Sigma k_{z}^{i} l_{i} ; \\
& k_{\psi \varphi}=\Sigma k_{z}^{i} l_{i} b_{i} ; k_{\psi \theta}=\Sigma k_{y}^{i} l_{i} h_{i} ; k_{\varphi \theta}=\Sigma k_{x}^{i} b_{i} h_{i},
\end{aligned}
$$

which depend on the stiffness and locating of the supporting surfaces.

During the machining of the prismatic workpiece with the center of weight $\mathrm{C}(\mathrm{L} / 2, \mathrm{~B} / 2, \mathrm{H} / 2)$ for locating chart 3 2-1 in three planes the stiffness coefficients make

$$
\begin{aligned}
& k_{x x}=\frac{1}{2} k_{y y}=k_{2} ; \\
& k_{z z}=3 k_{1} ; \\
& k_{\psi \psi}=\frac{1}{2} k_{1}(B-2 a)^{2} ; \\
& k_{\varphi \varphi}=\frac{3}{4} k_{1}(L-2 a)^{2} ; \\
& k_{\theta \theta}=\frac{1}{2} k_{2}(L-2 a)^{2} ; \\
& k_{z \varphi}=-\frac{1}{2} k_{1}(L-2 a),
\end{aligned}
$$

and another 8 coefficients of the cross stiffness make zero.

In order to consider the stiffness of the cutting tool and clamping element, the coefficient of stiffness of the clamping element $\mathrm{k}_{3}$ should be taken into account, and also the coefficients of longitudinal (along the axis $\mathrm{z}$ ), transverse (along the axes $\mathrm{x}, \mathrm{y}$ ) and angular (around the axis z) stiffness of the cutting tool (an example is end mills), which could be roughly estimated by the following approximate dependencies that determine the appropriate state of stress (compression, bending and torsion):

$$
k_{p}=\frac{\pi d^{2} E}{4 L_{p}} ; \quad k_{p}^{\prime}=\frac{3 \pi d^{4} E}{32 L_{p}^{3}} ; \quad k_{p}^{\prime \prime}=\frac{\pi d^{4} E}{64(1+v) L_{p}},
$$

where $d, L_{p}-$ diameter and length of the mill; E, $v-$ elasticity modulus of the first grade and Poisson's ratio of mill's material.

The consolidated coefficients of the direct and cross rigidities with the consideration of rigidities of the cutting instruments and clamping device receive the following form:

$$
\begin{aligned}
& k_{x x}=k_{2}+k_{p}^{\prime} ; \quad k_{y y}=2 k_{2}+k_{p}^{\prime} ; \quad k_{z z}=3 k_{1}+k_{3} ; \\
& k_{y \mu}=\frac{1}{2} k_{1}(B-2 a)^{2}+k_{p}\left(\frac{B}{2}-Y\right)^{2}+k_{p}^{\prime}\left(H_{1}-\frac{H}{2}\right)^{2} ; \\
& k_{\varphi \varphi}=\frac{3}{4} k_{1}(L-2 a)^{2}+k_{p}\left(\frac{L}{2}-X\right)^{2}+k_{p}^{\prime}\left(H_{1}-\frac{H}{2}\right)^{2} ; \\
& k_{\theta \theta}=\frac{1}{2} k_{2}(L-2 a)^{2}+k_{p}^{\prime}\left[\left(\frac{L}{2}-X\right)^{2}+\left(\frac{B}{2}-Y\right)^{2}\right]+k_{p}^{\prime \prime} ; \\
& k_{x \varphi}=-k_{p}^{\prime}\left(H_{1}-\frac{H}{2}\right) ; k_{x \theta}=-k_{p}^{\prime}\left(\frac{B}{2}-Y\right) ; \\
& k_{y \psi}=k_{p}^{\prime}\left(H_{1}-\frac{H}{2}\right) ; \\
& k_{y \theta}=-k_{p}^{\prime}\left(\frac{L}{2}-X\right) ; k_{z \psi}=-k_{p}\left(\frac{B}{2}-Y\right) ; \\
& k_{z \varphi}=-\frac{1}{2} k_{1}(L-2 a)-k_{p}\left(\frac{L}{2}-X\right) ; \\
& k_{\psi \varphi \varphi}=k_{p}\left(\frac{L}{2}-X\right)\left(\frac{B}{2}-Y\right) ; \\
& k_{\psi \theta}=-k_{p}^{\prime}\left(\frac{L}{2}-X\right)\left(H_{1}-\frac{H}{2}\right) ; \\
& k_{\varphi \theta}=-k_{p}^{\prime}\left(\frac{B}{2}-Y\right)\left(H_{1}-\frac{H}{2}\right) .
\end{aligned}
$$

During the cutting process, the presented coefficients of stiffness change according to the value, among them 4 - change its sign because of change of the coordinate $\mathrm{Y}$ of the location of cutting tool.

For free oscillations of the mechanical system "fixture - workpiece", the solution of the equation (9) is a harmonic movement described by the following statement: 


$$
\{X\}=\left\{X_{a}\right\} \sin \omega t,
$$

which includes an unknown own frequency $\omega$, and also vector-column of the peak values of displacements and rotation angles around the local system of coordinates $\left\{\mathrm{X}_{\mathrm{a}}\right\}=\left\{\mathrm{x}_{\mathrm{Ca}}, \mathrm{y}_{\mathrm{Ca}}, \mathrm{z}_{\mathrm{Ca}}, \varphi_{\mathrm{a}}, \psi_{\mathrm{a}}, \theta_{\mathrm{a}}\right\}^{\mathrm{T}}$. The last determines the form of free system oscillations with the accuracy up to the constant multiplier.

Equation (9) with the consideration of the statements (10) receives the following form:

$$
\left([K]-\omega^{2}[M]\right)\left\{X_{a}\right\}=\{0\} .
$$

The condition of existing of the nontrivial solutions of equation (18) allows recording the frequency equation:

$$
\left|[K]-\omega^{2}[M]\right|=0
$$

from which 6 eigenfrequencies of the mechanical system are determined:

$$
\begin{aligned}
& \omega_{1}=0 \sqrt{\frac{k_{2}}{m}} ; \quad \omega_{2}=\sqrt{\frac{2 k_{2}}{m}} ; \\
& \omega_{3}=\sqrt{\frac{3 k_{1}}{2}\left\{\frac{1}{m}+\frac{(L-2 a)^{2}}{4 J_{C_{y}}}-\sqrt{\left.\left[\frac{1}{m}+\frac{(L-2 a)^{2}}{4 J_{C y}}\right]^{2}-\frac{8(L-2 a)^{2}}{9 m J_{C y}}\right\}}\right.} \\
& \omega_{4}=(B-2 a) \sqrt{\frac{k_{1}}{2 J_{C x}}} ; \\
& \omega_{5}=\sqrt{\frac{3 k_{1}}{2}\left\{\frac{1}{m}+\frac{(L-2 a)^{2}}{4 J_{C y}}+\sqrt{\left[\frac{1}{m}+\frac{(L-2 a)^{2}}{4 J_{C y}}\right]^{2}-\frac{8(L-2 a)^{2}}{9 m J_{C y}}}\right\}} ; \\
& \omega_{6}=(L-2 a) \sqrt{\frac{k_{2}}{2 J_{C z}}} .
\end{aligned}
$$

Under condition (19) the equation (18) has the nontrivial solutions, which determine 6 forms of free oscillations with the accuracy up to the constant multiplier:

$$
\left\{\begin{array}{c}
y_{C}^{\langle n\rangle} \\
z_{C}^{\langle n\rangle} \\
\varphi^{\langle n\rangle} \\
\psi^{\langle n\rangle} \\
\theta^{\langle n\rangle}
\end{array}\right\}=-\left[d_{i, j}^{\langle n\rangle}\right]\left[\begin{array}{l}
K_{2,1}-\omega_{n}^{2} M_{2,1} \\
K_{3,1}-\omega_{n}^{2} M_{3,1} \\
K_{4,1}-\omega_{n}^{2} M_{4,1} \\
K_{5,1}-\omega_{n}^{2} M_{5,1} \\
K_{6,1}-\omega_{n}^{2} M_{6,1}
\end{array}\right\},
$$

where $\left[d_{i, j}^{\langle n\rangle}\right]$ - the main diagonal minor of the matrix of dynamic stiffness [D], which elements

$$
d_{i, j}^{\langle n\rangle}=K_{i, j}-\omega_{n} M_{i, j}, \quad(i, j=2,3, \ldots, 6) .
$$

are determined for the definite eigenfrequency $\omega_{\mathrm{n}}$ $(\mathrm{n}=1,2, \ldots, 6)$.

\subsection{Research of the forced oscillations}

Spatial motion of the workpiece in fixture as a body with 6 degrees of freedom in projections on the axes of the local system of coordinate xyz has the following form of equation (23), which could be presented in matrix form:

$$
[M]\left\{\ddot{X}_{C}\right\}+[K]\{X\}=\{F\},
$$

where $\{\mathrm{X}\}-$ vector-column of an unknown displacements; $\{\mathrm{F}\} \quad$ - vector-column of external dynamical action:

$$
\{X\}=\left\{\begin{array}{c}
x_{C} \\
y_{C} \\
z_{C} \\
\varphi \\
\psi \\
\theta
\end{array}\right\} ; \quad\{F\}=\left\{\begin{array}{c}
F_{x} \\
F_{y} \\
F_{z} \\
M_{x} \\
M_{y} \\
M_{z}
\end{array}\right\} .
$$

Herewith, the moments of dynamical components of cutting forces regarding axes of the local system of coordinates (Fig. 2 b):

$$
\begin{aligned}
& M_{x}=F_{y} h_{A}+F_{z} b_{A} ; \\
& M_{y}=F_{x} h_{A}+F_{z} l_{A} ; \\
& M_{z}=-F_{x} b_{A}+F_{y} l_{B} .
\end{aligned}
$$

Consider the case of monoharmonic external action:

$$
\{F\}=\left\{F_{a}\right\} \sin \omega t,
$$

where $\left\{F_{a}\right\}$ - vector-column of amplitudes of external forces and moments; $\omega=\omega_{0} Z-$ frequency, which is equal to the product of rotation frequency of the spindle and number of teeth $\mathrm{z}$ of the cutting tool.

Solution of equation (23) is sought in the form

$$
\{X\}=\left\{X_{a}\right\} \sin \omega t .
$$

For vector-column $\left\{X_{a}\right\}$, elements of which are displacement amplitudes of center of weight and rotation angles around the axes of coordinates.

Substitution of the statement (27) into the equation (23) allows writing down the equation

$$
\left([K]-\omega^{2}[M]\right)\left\{X_{a}\right\}=\left\{F_{a}\right\},
$$

the solution of which is

$$
\left\{X_{a}\right\}=\left([K]-\omega^{2}[M]\right)^{-1}\left\{F_{a}\right\} .
$$

Dependence of the component $X_{a i}$ of vector-column $\left\{X_{a}\right\}$ on frequency of external action $\omega$ determines the frequency-response characteristics of the mechanical system «fixture - workpiece». 
For locating chart 3-2-1 according to 3 planes frequency values of displacements of the center of weight of workpiece and rotation angles around the axes of coordinates have the following form:

$$
\begin{aligned}
& x_{C a}(\omega)=\frac{F_{x a} / m}{\omega_{1}^{2}-\omega^{2}} ; \\
& y_{C a}(\omega)=\frac{F_{y a} / m}{\omega_{2}^{2}-\omega^{2}} ; \\
& z_{c a}(\omega)=\frac{\left(\frac{k_{\varphi \varphi}}{\left.J_{C y}-\omega^{2}\right)}\right) \frac{F_{z a}}{m}-\frac{k_{z \varphi}}{J_{C y}} \frac{M_{z a}}{m}}{\left.\psi_{a}^{2}-\omega^{2}\right)\left(\omega_{5}^{2}-\omega^{2}\right)} \\
& \varphi_{a}(\omega)=\frac{M_{x a} / J_{C x}}{\omega_{4}^{2}-\omega^{2}} ; \\
& k_{z \varphi} \frac{F_{z a}}{J_{C y}}+\left(\frac{k_{z z}}{m}-\omega^{2}\right) \frac{M_{z a}}{J_{C y}}
\end{aligned}
$$

where $\omega_{1}, \omega_{2}, \ldots, \omega_{6}-$ value of eigenfrequencies, determined because of modal analysis.

According to the formula of passing from displacement of the center of weight of workpiece to displacement of supports

$$
\begin{aligned}
& x_{i}=x_{C}-b_{i} \theta+h_{i} \varphi \\
& y_{i}=y_{C}+l_{i} \theta+h_{i} \psi \\
& z_{i}=z_{C}+l_{i} \varphi+b_{i} \psi
\end{aligned}
$$

the frequency-response characteristics for supporting points have the following form:

$$
\begin{aligned}
& x_{i a}=\frac{F_{x a} / m}{\omega_{1}^{2}-\omega^{2}}+h_{i} \frac{-\frac{k_{z \varphi}}{J_{C y}} \frac{F_{z a}}{m}+\left(\frac{k_{z z}}{m}-\omega^{2}\right) \frac{M_{z a}}{J_{C y}}}{\left(\omega_{3}^{2}-\omega^{2}\right)\left(\omega_{5}^{2}-\omega^{2}\right)}- \\
& -b_{i} \frac{M_{z a} / J_{C z}}{\omega_{6}^{2}-\omega^{2}} ; \\
& y_{i a}=\frac{F_{y a} / m}{\omega_{2}^{2}-\omega^{2}}+h_{i} \frac{M_{x a} / J_{C x}}{\omega_{4}^{2}-\omega^{2}}+l_{i} \frac{M_{z a} / J_{C z}}{\omega_{6}^{2}-\omega^{2}} ; \\
& z_{i a}=\frac{\left(\frac{k_{\varphi \varphi}-k_{z \varphi} l_{i}}{J_{C y}}-\omega^{2}\right) \frac{F_{z a}}{m}+\left(\frac{k_{z z}-k_{z \varphi} / l_{i}}{m}-\omega^{2}\right) \frac{M_{z a} l_{i}}{J_{C y}}}{\left(\omega_{3}^{2}-\omega^{2}\right)\left(\omega_{5}^{2}-\omega^{2}\right)}+ \\
& +b_{i} \frac{M_{x a} / J_{C x}}{\omega_{4}^{2}-\omega^{2}} .
\end{aligned}
$$

So, for locating chart 3-2-1 according to three planes the location of mill $\mathrm{Y}=\mathrm{B} / 2$ the frequency-response characteristics of the system "fixture - workpiece" in the bottom supporting points have the following form:

$$
\begin{aligned}
& z_{1 a}=z_{2 a}=\frac{0,5 k_{1}(L-2 a)^{2}-J_{C y} \omega^{2}}{\left(\omega_{3}^{2}-\omega^{2}\right)\left(\omega_{5}^{2}-\omega^{2}\right)} \frac{F_{z a}}{m J_{C y}} ; \\
& z_{3 a}=\frac{k_{1}(L-2 a)^{2}-J_{C y} \omega^{2}}{\left(\omega_{3}^{2}-\omega^{2}\right)\left(\omega_{5}^{2}-\omega^{2}\right)} \frac{F_{z a}}{m J_{C y}} .
\end{aligned}
$$

The analysis of the last dependencies confirms that frequencies

$$
\begin{aligned}
& \omega^{\prime}=(L-2 a) \sqrt{\frac{k_{1}}{2 J_{C y}}} ; \\
& \omega^{\prime \prime}=(L-2 a) \sqrt{\frac{k_{1}}{J_{C y}}}=\frac{\omega^{\prime}}{\sqrt{2}} ;
\end{aligned}
$$

correspond the appearance of the antiresonance effect, for which zero value of amplitudes $\mathrm{z}_{1 \mathrm{a}}, \mathrm{z}_{2 \mathrm{a}}, \mathrm{i} \mathrm{z}_{3 \mathrm{a}}$ are is observed.

\section{Results and Discussion}

\subsection{Input data}

The verification of adequacy of the proposed mathematical model is carried out on the example of calculation of the mechanical system "fixture workpiece" for the locating chart on three planes. The prismatic workpiece with the overall dimensions of $100 \times 50 \times 50 \mathrm{~mm}$ is chosen for further research. Milling of the shoulder is investigated with the width $18 \mathrm{~mm}$ and cutting depth $5 \mathrm{~mm}$. The material of the workpiece is steel C45. The cutting tool is the solid end mill RF $100 \mathrm{VA}$ DIN $6527 \mathrm{~K}$ with the following parameters:

- diameter: $20 \mathrm{~mm}$;

- length of the cutting part: $42 \mathrm{~mm}$;

- total length: $92 \mathrm{~mm}$;

- number of teeth: 4;

- material: hard alloy.

The calculated results are:

- feed per tooth: $0.05 \mathrm{~mm} /$ tooth;

- feed per revolution: $0.2 \mathrm{~mm} / \mathrm{rev}$;

- cutting speed: $154 \mathrm{~mm} / \mathrm{min}$;

- rotating speed of the spindle: $2450 \mathrm{rpm}$. 


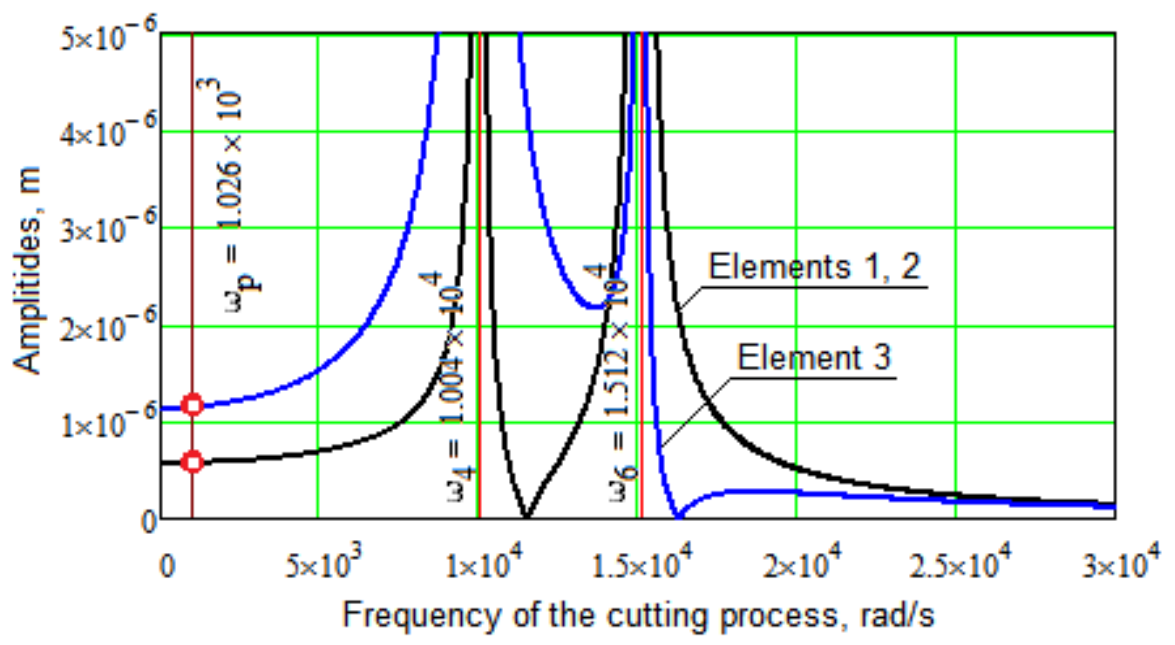

a

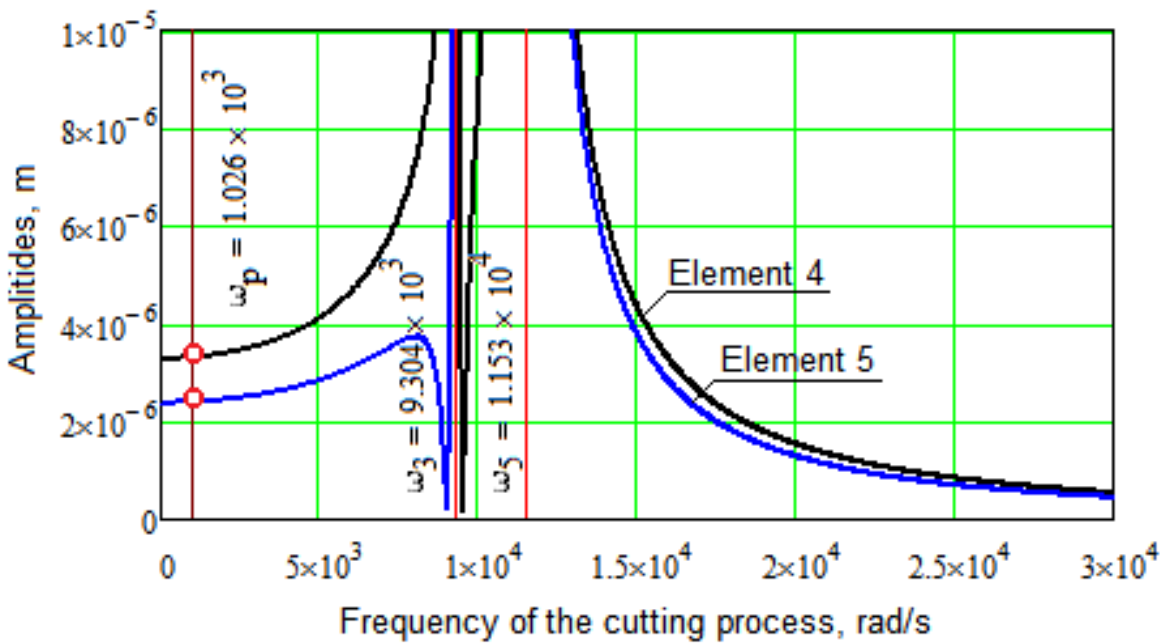

b

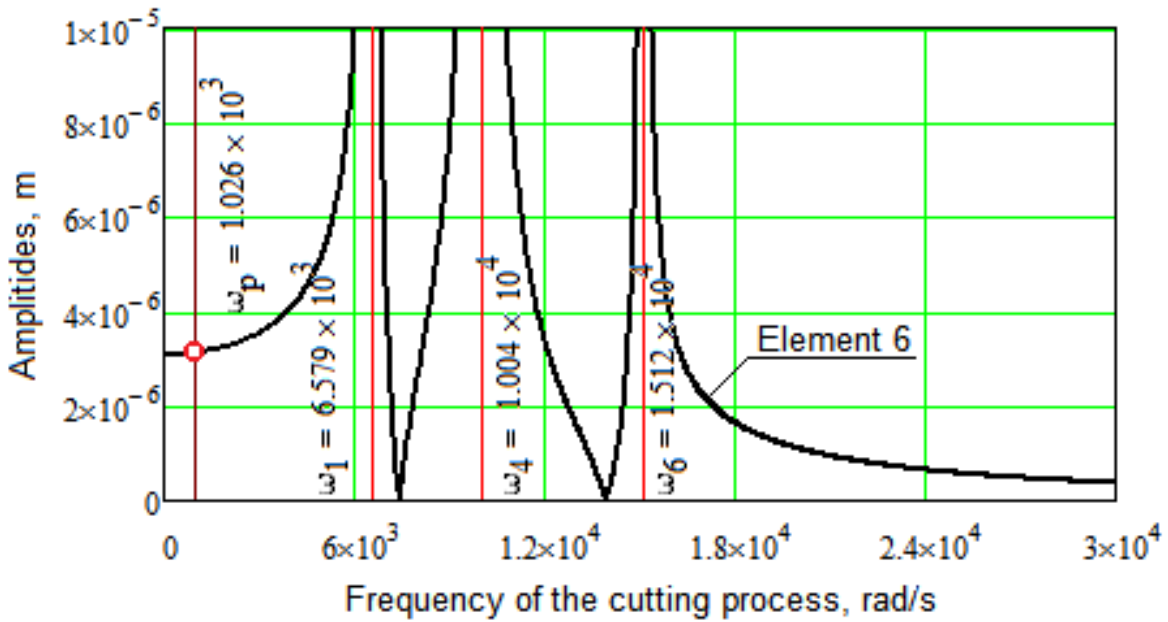

C

Figure 4. Amplitude-frequency response characteristics of functional elements oscillations: $a-$ for elements $1-3$; b- for elements $4-5$; $c-$ for element 6 
The abovementioned results correspond to the following cutting loads:

- components of the cutting force, N: $P_{z}=955$, $P_{y}=381, P_{x}=478$

- cutting torque, $\mathrm{N} \cdot \mathrm{m}: M_{z}=95.5$.

The workpiece is located on 6 supports, the stiffness of which is equal to $8.4 \cdot 10^{7} \mathrm{~N} / \mathrm{m}$.

\subsection{Calculation results}

Due to numerical calculations the following data are obtained:

- minimal value of the clamping force: $2.2 \mathrm{kN}$;

- mass of the workpiece: $1.95 \mathrm{~kg}$;

- axial moments of inertia: $J_{C x}=8.1 \cdot 10^{-4} \mathrm{~kg} \cdot \mathrm{m}^{2}$, $J_{C y}=J_{C z}=2.0 \cdot 10^{-3} \mathrm{~kg} \cdot \mathrm{m}^{2}$;

- operating frequency of the cutting process: 1026 $\mathrm{rad} / \mathrm{s}$;

- critical frequencies in ascending order, $\mathrm{rad} / \mathrm{s}: \omega_{1}=$ $6.6 \cdot 10^{3}, \omega_{2}=6.8 \cdot 10^{3}, \omega_{3}=9.3 \cdot 10^{3}, \omega_{4}=1.0 \cdot 10^{4}$, $\omega_{5}=1.2 \cdot 10^{4}, \omega_{6}=1.5 \cdot 10^{4}$.

The lowest critical frequency is 6.4 times higher than the operating frequency of the cutting process.

Values of the dynamic components of cutting forces $\left(F_{z a}=0.2 \cdot P_{z}=191 \mathrm{~N}, \quad F_{y a}=0.2 \cdot P_{y}=76 \mathrm{~N}\right.$, $\left.F_{x a}=0.2 \cdot P_{x}=96 \mathrm{~N}\right)$ and cutting torque $\left(M_{z a}=0.2 \cdot M_{z}=\right.$ $19 \mathrm{~N} \cdot \mathrm{m}$ ) allow determining amplitudes of oscillations of the functional elements at the operating frequency.

The obtained values of amplitudes (m) of the functional elements are:

- $z_{1 a}=z_{2 a}=5.7 \cdot 10^{-7}, z_{3 a}=1.1 \cdot 10^{-6}$ for elements that realized the primary datum;

- $y_{4 a}=3.3 \cdot 10^{-6}, y_{5 a}=2.4 \cdot 10^{-6}$ for elements of the secondary datum;

- $x_{6 a}=3.1 \cdot 10^{-6}$ for elements of the tertiary datum.

The quantitative indicators of the obtained results are relevant to the results of experimental research and do not exceed tolerances for the manufacture of corresponding parts for given production conditions.

\section{Conclusions}

The study of the previous experience, generalization and systematization of the existing approaches to the research of mechanical systems allowed making a proposal on development a new approach, which is the comprehensive analysis of equilibrium state and oscillations relatively fixed position of the workpiece as an element of the mechanical system with considering the stiffness of the cutting tool.

The mathematical model of static calculation system "fixture - workpiece" is being proposed, which ensures the defining of contact points of functional elements of fixture with the workpiece, the minimum required clamping force, components of cutting forces and places of application of these forces. Regression dependences for estimation of stiffness of functional elements of machine devices are being proposed.

The mathematical model of dynamic analysis, which is described by the system of differential equations of the 12 orders in matrix form relatively 6 variables in time independent parameters has been developed. The presented model allows research the eigenfrequencies of the system "fixture - workpiece" and determines the appropriate forms of free oscillations. The model also allows research the forced oscillations of the workpiece relatively the balanced position because of the action of dynamic component forces and cutting moments, and also determines frequency-response characteristics and the frequencies of resonance and antiresonance appearance.

The adequacy of the proposed mathematical model is approved by the example of numerical calculations. It is determined that the first critical frequency of oscillations of the workpiece is 6.4 times higher than the operating frequency of the cutting process, and the amplitude of forced oscillations is in a range of tolerances for the manufacture of the corresponding part.

The following researches are directed on the development of the methodology of calculation of the system "fixture - workpiece" by computer means of numerous realization and development of practical recommendations regarding its use during fixture design.

\section{Acknowledgement}

This research was partially funded by the Ministry of Education and Science of Ukraine within research projects "Integrated Technology of Modular Tooling Design" (State Registration Number 0117U002252) and "Development and Implementation of Energy-Efficient Modular Separation Devices for Oil and Gas Equipment” (State Registration Number 0117U003931).

\section{References}

[1] KARPUS, V.E. et al. (2012) Intensification of Manufacturing Processes (Sumy: Sumy State University, doi: 10.13140/2.1.3953.7604 [in Ukrainian].

[2] BI, Z.M. and ZHANG, W.J. (2001) Flexible Fixture Design and Automation: Review, Issues and Future Directions. International Journal of Production Research 39: 2867-2894, doi: 10.1080/00207540110054579.

[3] KARPUS, V.E. and IVANOV V.A. (2012) Choice of the Optimal Configuration of Modular Reusable Fixtures. Russian Engineering Research 32(3): 213-219, doi: 10.3103/S1068798X12030124

[4] NIXON, F. (1971) Managing to Achieve Quality and Reliability (Maidenhead: McGraw Hill).

[5] RONG, Y. and ZHU, Y. (1991) Computer-Aided Fixture Design (New York, Marcel Dekker).

[6] KUMBHAR, N. et al. (2012) Finite Element Modelling and Analysis of Workpiece - Fixture System. International Journal of Applied Research in Mechanical Engineering 2(2): 60-65. 
[7] LIAO, Y. and Hu, S. (2001) An Integrated Model of a Fixture - Workpiece System for Surface Quality Prediction. International Journal of Advanced Manufacturing Technology 17(11) 810-818, doi: 10.1007/s001700170108.

[8] KANG, Y. et al. (2003) Computer-Aided Fixture Design Verification. Part 3. Stability Analysis. The International Journal of Advanced Manufacturing Technology 21(10) 842-849, doi: 10.1007/s00170-002-1401-4.

[9] ASANTE, J. N. (2010) Effect of Fixture Compliance and Cutting Conditions on Workpiece Stability. The International Journal of Advanced Manufacturing Technology 48(1) 33-43, doi: 10.1007/s00170-009-2284-4.

[10] CIOATA, V. and KISS, I. (2009) The Machining Error due to Contact Deformation of Workpiece - Fixture System. Acta Technica Corviniensis - Bulletin of Engineering 2(1) 33-36.

[11] ZHENG, Y. (2005) Finite Element Analysis for Fixture Stiffness. PhD Thesis. (Worcester: Worcester Polytechnic Institute).

[12] ASADA, H. and BY, A. (1985) Kinematic Analysis of Workpart Fixturing for Flexible Assembly with Automatically Reconfigurable Fixtures. IEEE Journal on Robotics and Automation 1(2) 86-94, doi: 10.1109/JRA.1985.1087007.

[13] RONG, Y. and BAI, Y. (1997) Automated Generation of Fixture Configuration Design. Journal of Manufacturing Science and Engineering 119(2) 208-219, doi: 10.1115/1.2831097.

[14] CHOU, Y.C. (1993) Automated Fixture Design for Concurrent Manufacturing Planning. Concurrent Engineering 1(4) 219-229, doi: $10.1177 / 1063293 X 9300100405$.

[15] WU, Y. et al. (1997) Automated Generation of Dedicated Fixture Design. International Journal Computer Application in Technologies 10(3-4) 213-235, doi: 10.1504/IJCAT.1997.062249.

[16] TRAPPEY, A.J.C. et al. (1995) Computer-Aided Fixture Analysis Using Finite Element Analysis and Mathematical Optimization Modeling. Journal of Manufacturing Science and Engineering 2-1, 777-787.

[17] DENG, H. (2006) Analysis and Synthesis of Fixturing Dynamic Stability in Machining Accounting for Material Removal Effect. Ph.D. Thesis. (Atlanta: Georgia Institute of Technology).

[18] PAVLENKO, I.V. and IVANOV V.O. (2015) Ensuring of the Workpiece Stability Conditions in the Fixture with Locating Scheme on Three Planes. Bulletin of Sumy National Agrarian University 11(27) [in Ukrainian].

[19] IVANOV, V. et al. (2017) Numerical Simulation of the System "Fixture - Workpiece" for Levers Machining. The International Journal of Advanced Manufacturing Technology 91(1) 79-90, doi: 10.1007/s00170-016-9701-2.

[20] KARPUS, V.E. et al. (2012) Rapid-adjustable Locating Modules for Prismatic Parts. New Materials and Technologies in Metallurgy and Manufacturing 2, 91-94 [in Ukrainian]. 